\title{
Reaktion von Weisstannen auf ein- bis mehr- maligen Verbiss entlang von Lichtgradienten
}

Anna Camille Kolly

Andrea Doris Kupferschmid
Eidgenössische Forschungsanstalt für Wald, Schnee und Landschaft $(\mathrm{CH})$

Eidgenössische Forschungsanstalt für Wald, Schnee und Landschaft $(\mathrm{CH})^{*}$

\section{Response of Abies alba saplings to single and repeated browsing along light gradients}

\begin{abstract}
Browsing of silver fir (Abies alba) saplings by ungulates is one of the most important issues concerning decreasing regeneration of this tree species. In addition to genetic drivers, environmental factors such as light availability play an important role for the response of a browsed tree. Using an experimental design with fences, we investigated the influence of natural browsing on tree growth along a light gradient. The few fir saplings that responded by flagging up a lateral shoot compensated the browsing induced height difference compared to unbrowsed trees within four vegetation periods. Most firs however reacted with new shoots and remained smaller than unbrowsed ones. The more often a fir sapling was damaged on its terminal shoot, the smaller the tree remained. A positive relation between tree height and light was found only up to a canopy openness of $11 \%$. Mortality over the whole five years was high in low light $(<10 \%)$ and in forest gaps $(>15 \%)$, due to desiccation, competition with other plants or browsing ( $50 \%$ of total mortality). We recommend regenerating firs under moderately shaded conditions.
\end{abstract}

Keywords: Abies alba, ungulate browsing, growth rate, light gradient, tree regeneration, mammalian herbivory doi: $10.3188 /$ szf. 2014.0198

*Zürcherstrasse 111, CH-8903 Birmensdorf, E-Mail andrea.kupferschmid@wsl.ch
$\mathrm{D}$ ie Weisstanne (Abies alba Miller) ist in den Gebirgswäldern der Alpen infolge ihrer tiefen Pfahlwurzeln und ihrer geringen Anfälligkeit auf Insektenschädlinge im Vergleich zur Fichte eine sehr wichtige Baumart für die Gewährleistung der Schutzfunktion (Frehner et al 2005). Die Verbreitung dieser dritthäufigsten Baumart der Schweiz (Cioldi et al 2011) ist aber seit Längerem rückläufig (Tinner et al 2005). In den letzten Jahrzehnten wurde oft über ungenügende oder sogar fehlende Weisstannenverjüngung berichtet, insbesondere in den schweizerischen Jagdbanngebieten (Odermatt 2009). Dies erstaunt nicht, weil erstens die Dichten des Schalenwildes (Rothirsch, Reh und Gamswild) im Laufe des 20. Jahrhundert extrem zugenommen haben (nachdem diese Tierarten Ende des 19. Jahrhunderts bis auf Restpopulationen ausgerottet waren) und zweitens die Weisstanne zu den am meisten durch Schalenwild verbissenen Baumarten zählt (Kupferschmid \& Brang 2010).

Die Fähigkeit eines Baumes, auf Verbiss zu reagieren, wird nicht nur von den Genen gesteuert, sondern hängt auch stark von Umwelteinflüssen $a b$ (Häsler et al 2008, O’Reilly-Wapstraa et al 2014). Dies dürfte erklären, warum Eiberle in seinen Untersu- chungen zum Grenzwert für die zulässige Verbissintensität ${ }^{1}$ von Weisstannen Werte zwischen $5.2 \%$ und 19\% erhielt (Eiberle \& Dürr 1985, Eiberle \& Nigg 1987). Trotzdem wird heute in der Forstpraxis oft ein für die Beurteilung der Verbissbelastung standortunabhängiger Grenzwert von $9 \%$ verwendet. Im Triebschnittexperiment von Kupferschmid \& Bugmann (2013) konnten nach einmaligem Verlust des Endtriebs (Terminaltriebes) schnell wachsende, dominante Tannen den Höhenverlust durch Aufrichten eines Seitentriebes kompensieren und gar besser wachsen als unverbissene Bäumchen. Langsam wachsende (unterdrückte) Tannen bildeten jedoch insbesondere unter einer leichten künstlichen Beschattung erst neue Knospen und blieben damit klar kleiner (Kupferschmid \& Bugmann 2013). Im Gegensatz dazu kam Ammer (1996) beim Vergleich gezäunter und ungezäunter Flächen zum Schluss, dass unter Bedingungen, die auch ohne Verbiss kein üppiges Wachstum zulassen (Lichtmangel), sich der Verbiss nicht auswirkt, bei verbesserten Umweltbedingungen hingegen schon. An drei Standorten mit üppiger

\footnotetext{
1 Anteil am Endtrieb verbissener Pflanzen mit einer Höhe von 10 bis $130 \mathrm{~cm}$
} 
Tannennaturverjüngung beeinflusste das Licht das Wachstum nach Verbiss durch Rehe nur indirekt, indem Tännchen mit mehr Licht schneller eine Höhe erreichten, bei der sie mit Trieben aus vorhandenen Knospen reagieren konnten (Kupferschmid et al 2013). Die Reaktion und damit die Fähigkeit der Weisstanne, einen Baumhöhenverlust zu kompensieren, dürfte also vom vorhandenen Licht abhängen.

In dieser Studie soll abgeklärt werden, wie Weisstannenverjüngung auf natürlichen Schalen-
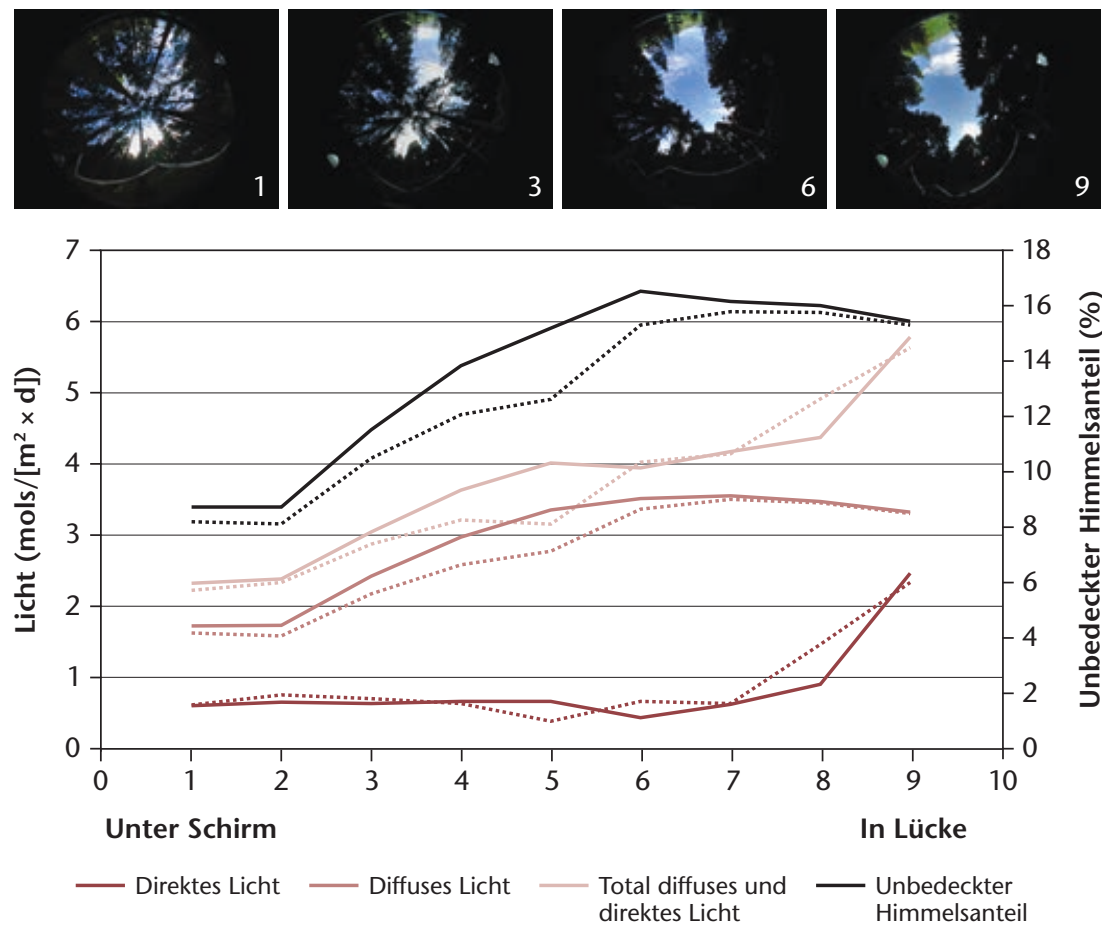

Abb 1 Verfügbares direktes und diffuses Licht sowie unbedeckter Himmelsanteil entlang des Lichtgradienten von unter Schirm bis in die Lücke im permanent eingezäunten Teil (ausgezogene Linien) und im zeitweise ausgezäunten Teil (gepunktete Linie) der Fläche A. Die Nummern bei den Fischaugenfotos entsprechen den Standortnummern entlang des Lichtgradienten ( $x$-Achse).

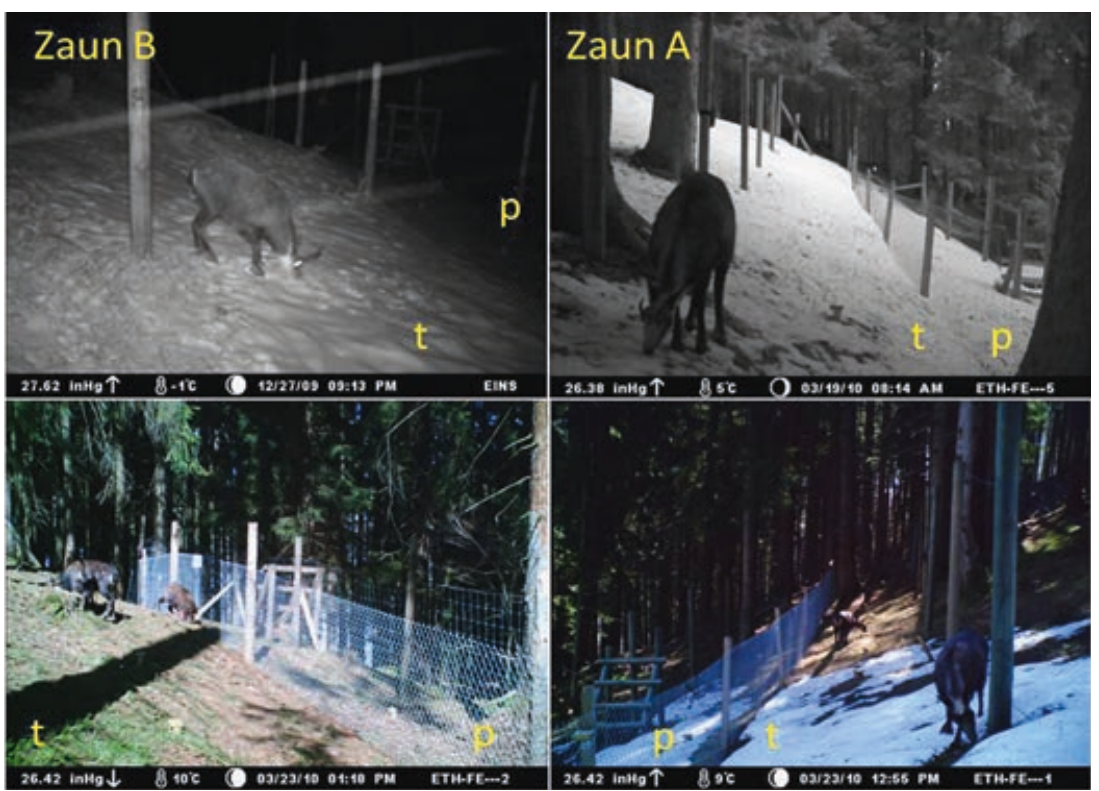

Abb 2 Wildkamerabilder der beiden Zäune zu Zeiten mit wenig bis keinem Schnee im Winter 2009/2010. t: zwischen 14. Oktober 2009 und 31. März (Zaun B) bzw. 20. April 2010 (Zaun A) temporär ausgezäunte Teilfläche, p: permanent gezäunte Teilfläche. wildverbiss reagiert und unter welchen Bedingungen sich die verbissenen Bäumchen vier Vegetationsperioden nach dem Verbiss bezüglich Baumhöhe und Höhenzuwachs erholen konnten. Insbesondere soll quantifiziert werden, inwieweit die Verfügbarkeit von Licht die Reaktion auf Verbiss beeinflusst und ob es einen Licht-Schwellenwert für die Reaktion gibt. Zusätzlich soll untersucht werden, wie die Weisstanne auf mehrfachen Verbiss reagiert und wann Mehrstämmigkeit entsteht.

\section{Methoden}

Im Altenbann-Wald (Koordinaten $46^{\circ} 59^{\prime} 00^{\prime \prime} \mathrm{N} /$ $9^{\circ} 05^{\prime} 07^{\prime \prime E}$ ) oberhalb von Schwanden (GL), an einem nordostexponierten Hang (55-76 $)$ auf etwa 995 bis 1020 m ü.M., wurde ein Feldexperiment mit gepflanzten Tannen durchgeführt. Dieser vor allem mit Fichten bestockte typische Waldhirsen-Buchenwald auf skelettreicher Braunerde gehört zu einem der ältesten Wildschutzgebiete Europas, dem Freiberg Kärpf, und beheimatet grosse Populationen von Rehen, Rothirschen und Gamswild (Odermatt 2009). Entlang eines Lichtgradienten von unter Schirm bis in kleine Baumlücken (Abbildung 1) wurden zwei $2 \mathrm{~m}$ hohe Zäune mit Maschenweite $10 \mathrm{~cm}$ errichtet: Zaun A umschliesst eine Fläche von $25 \mathrm{~m} \times 6 \mathrm{~m}$, Zaun B eine solche von $18 \mathrm{~m} \times 6 \mathrm{~m}$. Beide Zäune liegen parallel zu den Höhenlinien und wurden in der Mitte längs durch einen weiteren Zaun halbiert, sodass die obere Hälfte geöffnet und somit dem Wild zugänglich gemacht werden konnte (Abbildung 2). Die untere Hälfte blieb als Kontrolle während der ganzen Versuchsdauer geschlossen. Die Fläche A hat eine Neigung von $26^{\circ}$ unter Schirm bis $37^{\circ}$ in der Lücke, die Fläche B ist überall durchschnittlich $15^{\circ}$ geneigt. Eine weitere Versuchsfläche, die Fläche C, lag im geschlossenen Bestand zwischen den beiden Flächen A und B und war nie eingezäunt (4 Teilflächen à $2.5 \mathrm{~m} \times 2.5 \mathrm{~m}$ mit Neigung 7-35 $5^{\circ}$.

Alle $2 \mathrm{~m}$ wurden Fischaugenbilder zur Lichtmessung aufgenommen; daraus konnten der unbedeckte Himmelsanteil sowie die Menge an direktem und diffusem Licht bestimmt werden. Die Fläche A wies einen deutlich stärkeren Lichtgradienten (unbedeckter Himmelsanteil von 8.1 bis $16.5 \%$; Abbildung 1) als Fläche B (9.4 bis 13.2\%) auf, und Fläche $\mathrm{C}$ hatte einen unbedeckten Himmelsanteil von 8 bis $10 \%$.

Mitte Mai 2008 wurden 747 2-jährige Weisstannen im Abstand von $50 \mathrm{~cm}$ in die drei Flächen gepflanzt. Das Saatgut kam aus dem Lochwald oberhalb Oberhelfenswil (SG, 750 m ü.M., westexponiert). Im September 2008 wurden in den Flächen A und B insgesamt 56 Tännchen nachgepflanzt (51 als Ersatz von abgestorbenen und 5 ursprünglich vergessene). Im Herbst 2008 lebten in Fläche A 392 Tännchen, in 


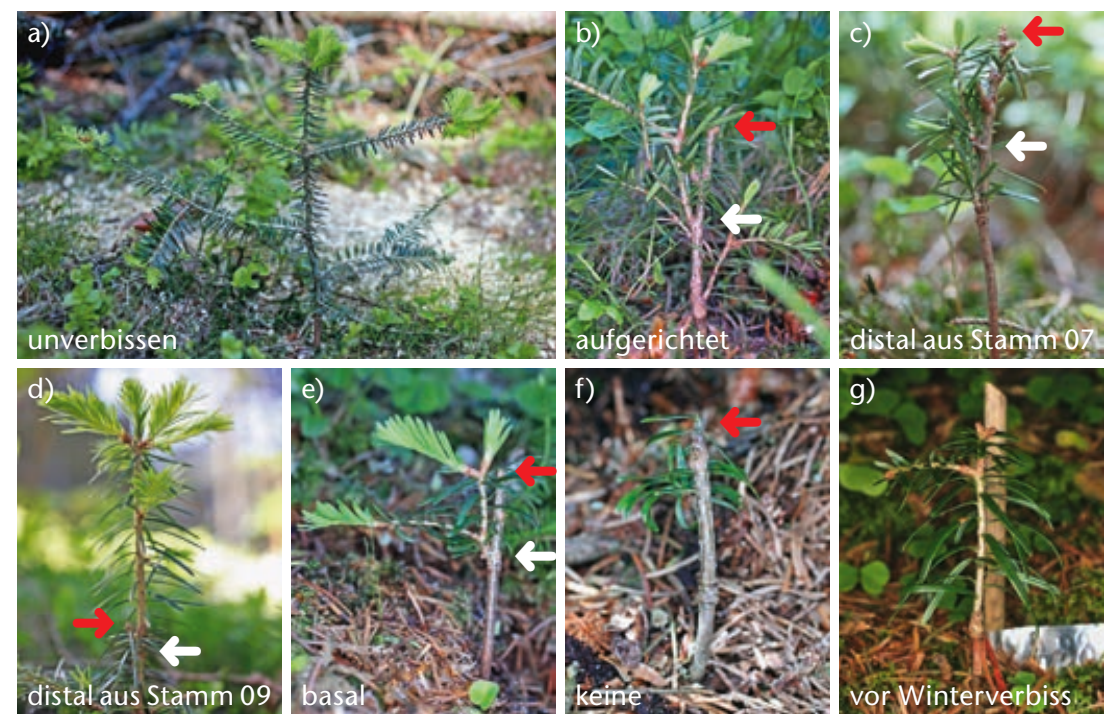

Abb 3 Beispiele unterschiedlicher Reaktionsarten von Weisstännchen im dritten Frühjahr nach Verbiss (Mai 2012): a) unverbissen, b) Aufrichten eines 2008 gebildeten «Kurztriebes» am Stammstück des Jahres 2007, c) distaler Trieb aus Stamm 2007, d) distaler Trieb aus Stamm 2009, e) basaler Trieb aus Quirl Keimling/2007, f) Bäumchen ohne neuen Trieb (= keine Reaktion). Das Foto $g$ ) zeigt das Bäumchen in $f$ ) ein Jahr vor Verbiss im Herbst 2008. Rote Pfeile: Verbiss im Winter 2009/2010, weisse Pfeile: Reaktionsstelle. Fotos: Ulrich Wasem/WSL von tiefer unten herauswuchs (Abbildungen 3c-d). Auch wurden die senkrecht wachsenden Endtriebe gezählt, um eine allfällige Aufgliederung der Stammachse zu erfassen (Mehrstämmigkeit).

Die statistischen Auswertungen wurden mit der Software R (Version 3.0.1) durchgeführt (R Core Team 2013). Wir berechneten lineare Regressionsmodelle für numerische Zielvariablen (Baumhöhe, Höhenzuwachs) mit der Funktion lm und für binomiale Variablen (Mortalität) mit der Funktion glm. Es wurden stets statistische Modelle mit dem tiefsten Akaike-Informationskriterium (AIC) gewählt (Methode analog zu Kapitel 5 in Stauffer 2008), und das Signifikanzlevel für die einzelnen Variablen/Faktoren betrug $\mathrm{p}<0.05$.

Die Daten bis Herbst 2011, also zwei Vegetationsperioden nach der Zaunöffnung und dem einmaligen Winterverbiss 2009/2010, wurden in Kupferschmid et al (2014) publiziert und werden hier jeweils nur kurz zusammengefasst. Im vorliegenden Beitrag werden die Resultate vier Vegetationsperioden nach der Zaunöffnung und besonders auch nach mehrmaligem Verbiss dargestellt und diskutiert.

Fläche B 278 und in Fläche C 82. Im Herbst 2008 und 2009 wurden jeweils die Baumhöhe, der jährliche Höhenzuwachs und die allfällige Todesursache aufgenommen.

Am 14. Oktober 2009 wurden die oberen Hälften der Flächen A und B geöffnet, um dem Wild Zugang zu den Tännchen zu verschaffen. Mit zwei bis drei Wildkameras pro Fläche filmten wir den Verbiss während der ganzen Phase der Zaunöffnung. Die Zäune wurden am 31. März (Zaun B) beziehungsweise 20. April 2010 (Zaun A) wieder ganz geschlossen (Unterschied wegen Schneedecke, Abbildung 2). Direkt danach ordneten wir bei jedem Tännchen den Verbiss dem Gamswild (rechtwinklig zur Triebachse abgebissene Triebe), den Mäusen (schräg zur Triebachse abgebissene Triebe inkl. sichtbarer Zahnspuren oder Rindenfrass) oder in unklaren Fällen «unbekannt» zu.

Unmittelbar nach der Zaunschliessung sowie ein, zwei und vier Vegetationsperioden danach wurden erneut die Höhe und der Höhenzuwachs jedes Tännchens gemessen. Zudem unterschieden wir folgende Reaktionsarten (Abbildung 3 und Zeichnungen in Abbildung 6): 1) «Aufrichten» eines bereits vor dem Verbiss vorhandenen Triebes (auch als «Aufstellen» bezeichnet), 2) «basal», wenn der neue Endtrieb aus einer Knospe an einem Astquirl entstand, 3) «distal», wenn der neue Trieb aus einer Knospe an einem Stamm zwischen Astquirlen austrieb (solche Knospen wären sonst zu «Kurztrieben» oder schlafenden Knospen geworden), oder 4) «keine» Reaktion, wenn seit dem Verbiss kein neuer senkrecht wachsender Endtrieb gebildet wurde. Des Weiteren wurde geprüft, ob der neue Endtrieb aus dem obersten noch vorhandenen Quirl/Reststück stammte oder

\section{Resultate}

\section{Verbiss}

Während der Zaunöffnung im Winter 2009/ 2010 wurden 59\% der im Sommer 2013 noch lebenden Tännchen in den nicht eingezäunten Flächen A und B verbissen. Wir filmten keine fressenden Rehe oder Hirsche, sondern ausschliesslich Gamswild, womit Verbiss durch Rehe oder Hirsche ausgeschlossen werden kann. Wie die Auswertung der Verbissspuren zeigte, verbiss in der Fläche B fast ausschliesslich Gamswild (98\%). In der Fläche A konnten hingegen rund 28\% der Frassspuren auf Mäuse und 53\% auf Gamswild zurückgeführt werden, während 19\% nicht klar zuweisbar waren. Mäuse konnten durch die groben Zaunmaschen auch in permanent eingezäunte Flächen eindringen und verbissen in Fläche A bis und mit Winter 2010/2011 8.5\% der Tännchen, in Fläche B hingegen keine.

Meist handelte es sich um starken Verbiss, da nicht nur der letztjährige Endtrieb, sondern auch weiter unten liegende Stammstücke und/oder Seitentriebe abgefressen wurden (Beispiele in Abbildung 3). Wildverbiss fand erst im Winter in Zeiten mit wenig oder keinem Schnee statt (Auswertung Wildkamera; Beispiel in Abbildung 2); damit korrelierte der Gamsverbiss signifikant negativ mit der Schneedauer und dem unbedeckten Himmelsanteil. Mausverbiss war hingegen signifikant häufiger bei mehr Licht, also besonders in der Lücke der Fläche A. Der Verbiss im Winter 2009/2010 war damit mehr oder weniger homogen über den gesamten Lichtgradienten verteilt. 
Obwohl in den folgenden Jahren die Zäune geschlossen blieben, konnte bei 18\% der Tännchen der Flächen A und B im Sommer 2013 weiterer Verbiss festgestellt werden. In den Wintern 2011/2012 und 2012/2013 wurden die Zäune der Fläche A und $B$ beide durch einen umgefallenen Baum beschädigt, wodurch die Flächen für kurze Zeit für das Wild zugänglich waren. Die Endtriebverluste von 2011 bis 2013 konnten anhand der Verbissspuren zur Hälfte

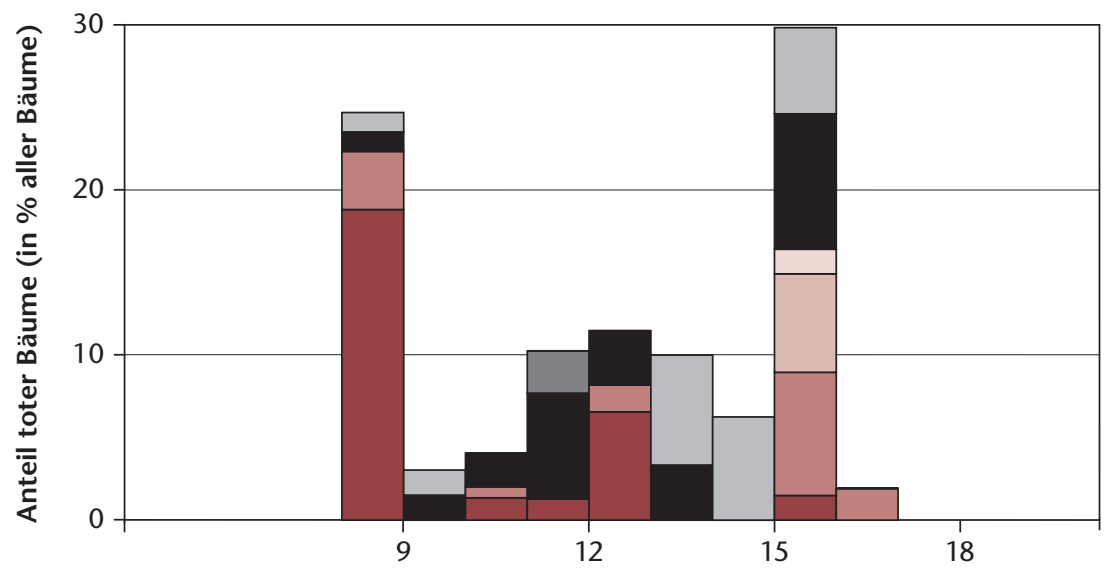

Unbedeckter Himmelsanteil (\%)

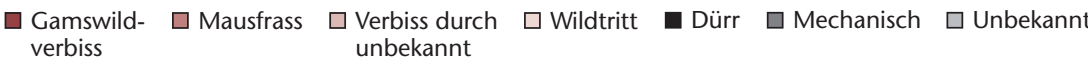

Abb 4 Anteil toter Weisstännchen im Sommer 2013 bezogen auf die Anzahl der im Herbst 2008 lebenden Weisstännchen nach Todesursache entlang des Lichtgradienten in den Flächen $A$ und B. Bei rund der Hälfte der toten Bäumchen war das Absterben höchstwahrscheinlich auf Tiere zurückzuführen.

\begin{tabular}{|c|c|c|c|c|c|c|}
\hline & \multicolumn{2}{|c|}{ Tot (\%) } & \multicolumn{2}{|c|}{ Lebend (\%) } & \multirow[b]{2}{*}{ Total (Anzahl) } \\
\hline & & Verbissen & Unverbissen & Verbissen & Unverbissen & \\
\hline \multirow{9}{*}{$\begin{array}{l}\frac{0}{U} \\
\frac{\pi}{4}\end{array}$} & A ohne Zaun & 20.1 & 7.6 & 46.7 & 25.6 & 184 \\
\hline & A mit Zaun & 1.0 & 0.5 & 30.9 & 67.6 & 191 \\
\hline & A total & 10.4 & 4.0 & 38.7 & 46.9 & 375 \\
\hline & B ohne Zaun & 3.6 & 1.2 & 64.5 & 30.7 & 166 \\
\hline & B mit Zaun & 0.0 & 0.0 & 9.8 & 90.2 & 102 \\
\hline & B total & 2.2 & 0.7 & 43.7 & 53.4 & 268 \\
\hline & $\mathrm{A}+\mathrm{B}$ ohne Zaun & 12.3 & 4.6 & 55.1 & 28.0 & 350 \\
\hline & $A+B$ mit Zaun & 0.7 & 0.4 & 23.5 & 75.4 & 293 \\
\hline & $A+B$ total & 7.0 & 2.6 & 40.8 & 49.6 & 643 \\
\hline \multirow{9}{*}{ 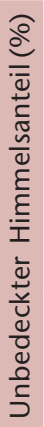 } & $8-9$ & 22.9 & 0.0 & 71.1 & 6.0 & 83 \\
\hline & $9-10$ & 1.8 & 0.0 & 29.2 & 69.2 & 65 \\
\hline & $10-11$ & 2.1 & 0.0 & 54.5 & 43.4 & 145 \\
\hline & $11-12$ & 2.7 & 1.4 & 39.7 & 56.2 & 73 \\
\hline & $12-13$ & 6.8 & 1.7 & 52.5 & 39.0 & 59 \\
\hline & $13-14$ & 0.0 & 6.9 & 27.6 & 65.5 & 29 \\
\hline & $14-15$ & 0.0 & 6.3 & 12.5 & 81.2 & 16 \\
\hline & $15-16$ & 14.0 & 8.3 & 25.6 & 52.1 & 121 \\
\hline & $16-17$ & 1.9 & 0.0 & 7.7 & 90.4 & 52 \\
\hline
\end{tabular}

Tab 1 Anteil toter beziehungsweise lebender Weisstännchen im Sommer 2013, welche zwischen Herbst 2009 und Sommer 2013 am Endtrieb verbissen wurden beziehungsweise unverbissen blieben, bezogen auf die Anzahl der im Herbst 2009 lebenden Bäumchen (Total). Zwei Bäumchen mit Todesursache Wildtritt sind in dieser Tabelle nicht aufgeführt. Beim Zusammenhang mit dem unbedeckten Himmelsanteil wurden die Flächen $A$ und $B$ zusammen analysiert. auf Mäuse und zu einem Drittel auf Wildverbiss zurückgeführt werden; bei einem Fünftel der Verbissspuren blieb der Verursacher unbekannt.

In der nie eingezäunten Fläche $C$ war der Endtrieb im Juli 2013 nur an vier der 23 noch lebenden Tännchen unverbissen. Bei einem davon war die Terminalknospe 2013 allerdings durch ein Insekt beschädigt worden. In dieser überschirmten Fläche wurde kein Mausfrass festgestellt.

\section{Mortalität}

Von den im Herbst 2008 in den Flächen A und B lebenden Tännchen starben bis Sommer 2013 rund 13\% (89 Pflanzen). Alleine im ersten Winter starben 25 Tännchen (4 davon waren Ersatzpflanzen), wovon alle bis auf eines wegen Trockenheit. Über die gesamten fünf Jahre war bei rund der Hälfte der toten Bäumchen das Absterben höchstwahrscheinlich auf Tiere zurückzuführen, d.h., sie wurden von Gamswild und Mäusen verbissen und wiesen sonst keine Schädigungen auf, und zwei Bäumchen wurden durch das Gamswild zertreten (Abbildung 4).

Betrachtet man nur die Zeit seit der temporären Zaunöffnung im Herbst 2009, so starben in den Flächen A und B 64 Tännchen ab, drei Viertel davon waren verbissen. Die Sterberate der Tännchen unterschied sich zwischen den Flächen A und B sowie zwischen Flächen mit und ohne Zaun. Am meisten tote Bäumchen wurden im ungezäunten Teil der Fläche A gefunden, während im eingezäunten Teil der Fläche B zwischen Herbst 2009 und Sommer 2013 kein Baum abstarb (Tabelle 1).

An den sehr dunklen (8-9\% unbedeckter Himmelsanteil) und den hellen Standorten (15-16\% unbedeckter Himmelsanteil) war die Sterblichkeit hoch und meist durch Tiere verursacht. Bei mittleren Lichtverhältnissen hingegen war die Sterblichkeit geringer (Abbildung 4 und Tabelle 1).

In der Fläche $\mathrm{C}$, welche den Tieren während sechs Jahren freien Zugang bot, lebten im Sommer 2013 nur noch 23 der ursprünglich 82 gepflanzten Tännchen, die Mortalität betrug somit 72\%. Hier waren knapp 70\% der abgestorbenen Bäume durch Gamswild verbissen. Rechnet man alle nicht mehr gefundenen Bäume und solche mit unklarer Todesursache hinzu, so kommt man auf 90\%, denn nur sechs waren zwischen Pflanzung im Frühling 2008 und Sommer 2013 vertrocknet.

Baumhöhe und Höhenzuwachs im Jahr 2013

Im Sommer 2013 waren die Tännchen der Flächen A und B durchschnittlich $24.6 \pm 10.7 \mathrm{~cm}$ (Mittelwert \pm Standardabweichung) hoch (Abbildung 5a, Minimum $3 \mathrm{~cm}$, Maximum $59 \mathrm{~cm}$ ). Am Endtrieb verbissene Bäumchen waren im Mittel $14.0 \pm 7.0$ cm kleiner als unverbissene. Der Höhenzuwachs 2013 betrug zwischen 0 und $15 \mathrm{~cm}$, durchschnittlich $5.0 \pm 3.1 \mathrm{~cm}$ (Abbildung 5d). Tännchen, welche nie einen End- 

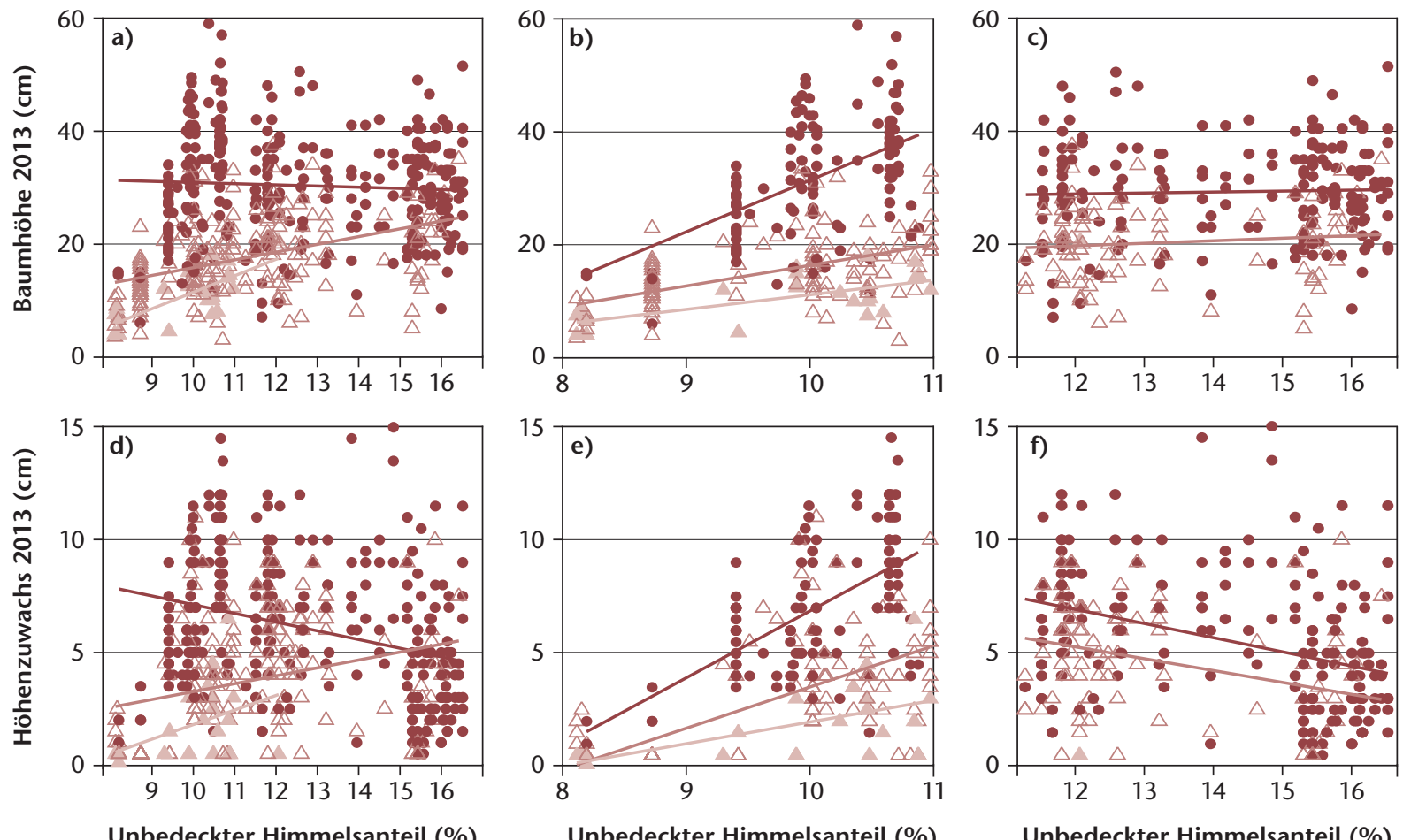

Unbedeckter Himmelsanteil (\%)

Unbedeckter Himmelsanteil (\%)

Unbedeckter Himmelsanteil (\%)

Endtriebschäden $\rightarrow$ Keine $\triangle$ Einer $\rightarrow$ Mehrere

Abb 5 Baumhöhe (oben) und Höhenzuwachs 2013 (unten) der Weisstännchen entlang des Lichtgradienten (unbedeckter Himmelsanteil) zusammen für die Flächen A und B. a und d: entlang des ganzen Lichtgradienten, b und e: nur bis zu einem unbedeckten Himmelsanteil von 11\%, c und f: ab einem unbedeckten Himmelsanteil von $11 \%$.

triebverlust erlitten, wuchsen 2013 durchschnittlich $6.2 \pm 3.1 \mathrm{~cm}$, Tännchen, die einzig im Winter 2009/ 2010 verbissen worden waren, $4.3 \pm 2.2 \mathrm{~cm}$ und solche, die 2011, 2012 oder 2013 das erste Mal einen Endtriebverlust erlitten, $2.3 \pm 3.1 \mathrm{~cm}$.

Die Anzahl Verluste am Endtrieb war für die Höhe des Baumes besonders wichtig: Je öfter der Endtrieb beschädigt wurde, desto kleiner blieb auch der Baum (Abbildung 5a). Entsprechend war bei einer höheren Anzahl Endtriebverluste auch der Höhenzuwachs kleiner (Abbildung 5d).

Je mehr Licht den Tännchen zur Verfügung stand, desto höher wuchsen sie, obwohl eine grosse Variabilität vorhanden war. Insbesondere hängen Baumhöhe und Licht bis $\mathrm{zu}$ einem unbedeckten Himmelsanteil von ca. 11\% positiv linear zusammen (Abbildung 5b). Bei mehr Licht war dies nicht der Fall (Abbildung 5c). Für das Wachstum des Endtriebes scheint die Relation mit zunehmendem Licht komplexer; es konnte unabhängig vom Verbiss kein

\begin{tabular}{|l|c|c|c|c|c|}
\hline Verbiss im: & Anzahl ver- & \multicolumn{4}{|c|}{ Reaktionsart (\%) } \\
\cline { 3 - 6 } & $\begin{array}{c}\text { Tissener } \\
\text { Tannen }\end{array}$ & $\begin{array}{c}\text { Basaler } \\
\text { Trieb }\end{array}$ & $\begin{array}{c}\text { Distaler } \\
\text { Trieb }\end{array}$ & $\begin{array}{c}\text { Aufgerich- } \\
\text { teter Trieb }\end{array}$ & $\begin{array}{c}\text { Kein neuer } \\
\text { Trieb }\end{array}$ \\
\hline Winter 2009/2010 & 154 & 70.8 & 20.2 & 4.5 & 4.5 \\
\hline $2011-2013$ & 71 & 80.3 & 11.3 & 1.4 & 7.0 \\
\hline mehrmals & 28 & 53.6 & 17.9 & 7.1 & 21.4 \\
\hline
\end{tabular}

Tab 2 Anzahl am Endtrieb einmal verbissene (entweder im Winter 2009/2010 während der temporären Zaunöffnung oder in den Jahren 2011 bis 2013) respektive mehrfach verbissene Weisstännchen und deren proportionaler Anteil je Reaktionsart in den Flächen A und $B$. linearer Zusammenhang nachgewiesen werden. Ein unbedeckter Himmelsanteil von circa 11\% (was 2.3 mols $/\left[\mathrm{m}^{2} \times \mathrm{d}\right]$ diffusem Licht mit $0.7 \mathrm{mols} /\left[\mathrm{m}^{2} \times \mathrm{d}\right]$ direktem Licht in Fläche A [Abbildung 1] respektive mit $2.5 \mathrm{mols} /\left[\mathrm{m}^{2} \times \mathrm{d}\right]$ direktem Licht in Fläche B entspricht) scheint aber auch beim Höhenzuwachs einen Schwellenwert zu bilden (Abbildungen 5e-f).

\section{Wie reagierten die Tannen auf den Verbiss im Winter 2009/2010?}

Die meisten der verbissenen, lebenden Tännchen in den Flächen A und B bildeten bis Sommer 2013 (also vier Vegetationsperioden seit der temporären Zaunöffnung im Winter 2009/2010) einen neuen Endtrieb aus basalen Knospen (Tabelle 2). Ein Fünftel der Tännchen bildete einen neuen Endtrieb aus einer distalen Knospe, und je $4.5 \%$ der Tännchen richteten einen Trieb auf respektive hatten immer noch keinen neuen Endtrieb. Eine ausbleibende Triebbildung (d.h. keine Reaktion) und tendenziell auch ein distaler Endtrieb aus tieferen Stammstücken (vgl. Abbildung 3c) waren zwei Jahre nach Verbiss typischer für beschattete Tännchen als für solche in Baumlücken. Im Sommer 2013 kamen hingegen alle Reaktionsarten über den ganzen Lichtgradienten in etwa gleich oft vor ( $\Delta$ in Abbildung 7 ). Dies bedeutet, dass Tännchen besonders im Schatten verzögert reagierten, also erst im dritten oder vierten Jahr nach Verbiss einen neuen Endtrieb bildeten.

Eine bis vier Vegetationsperioden nach dem Verbiss unterschieden sich unverbissene Tännchen 

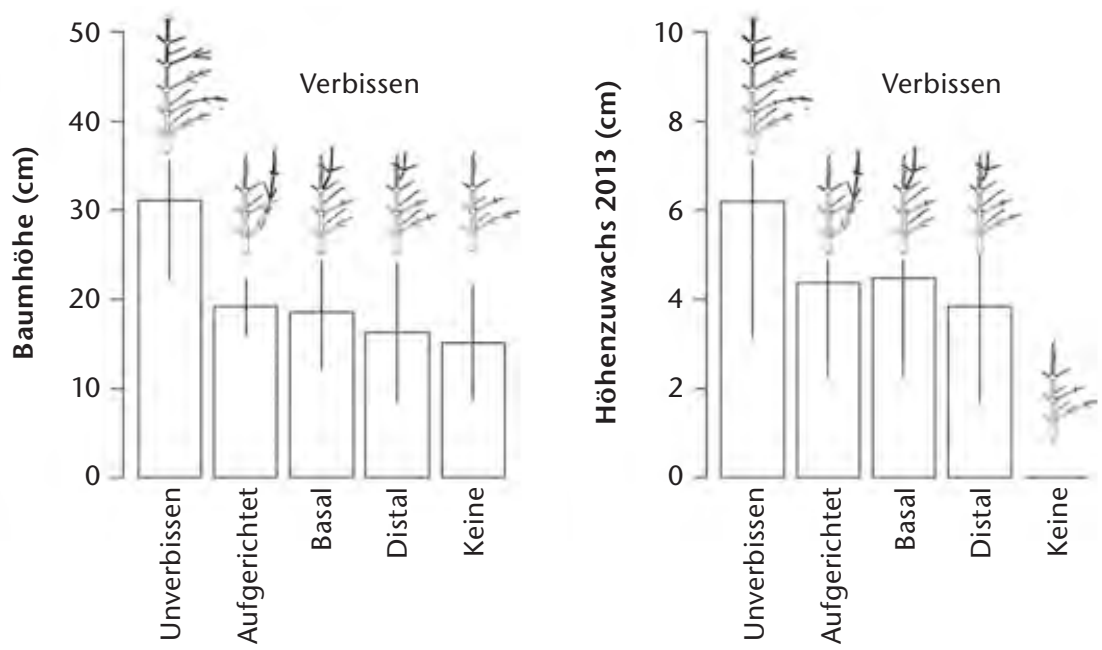

Abb 6 Baumhöhe und Höhenzuwachs 2013 mit Standardabweichung bei unverbissenen Weisstännchen beziehungsweise einmalig im Winter 2009/2010 verbissenen mit unterschiedlicher Reaktionsart. Die Zeichnungen stellen Beispiele im Zustand zwei Jahre nach dem im Winter 2009/2010 erfolgten Verbiss dar (gezeichnet von A. Schwyzer, WSL).

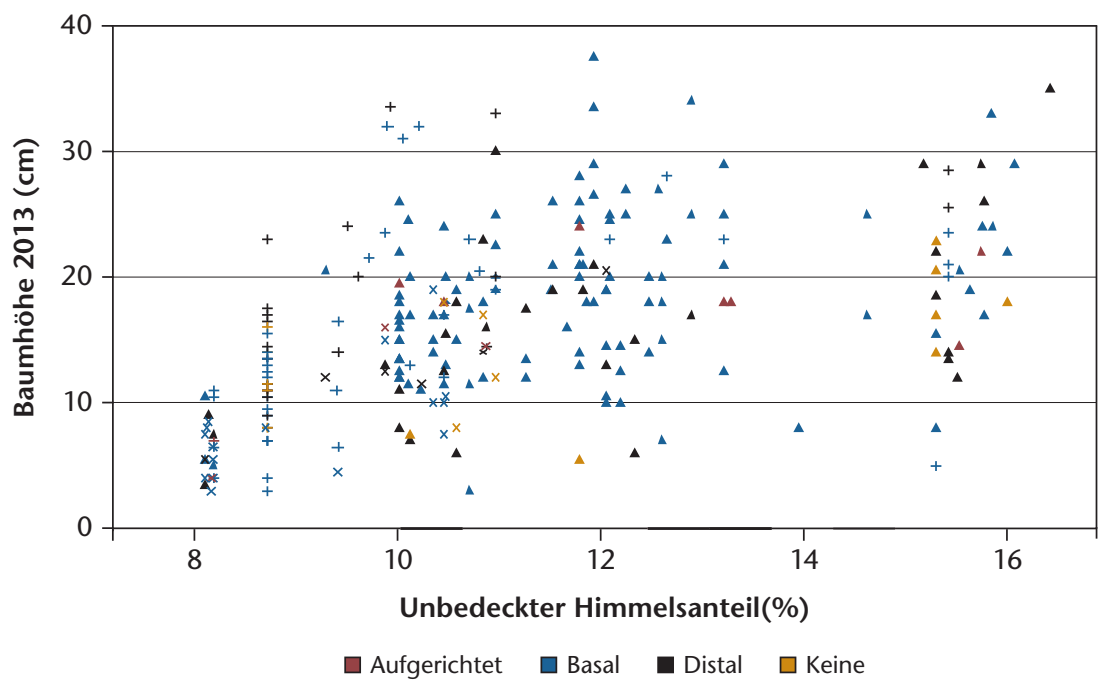

Abb 7 Baumhöhe 2013 für Weisstännchen, die nur im Winter 2009/2010 verbissen wurden ( $\Delta)$, für solche, die erstmals und einmalig im Jahr 2011, 2012 oder 2013 verbissen wurden (+), und für solche, die mehrmals verbissen wurden ( $\times$ ). Farben symbolisieren die unterschiedlichen Reaktionsarten.

und solche, die nach Verbiss mit dem Aufrichten eines Triebes reagiert hatten, in der Baumhöhe nicht signifikant (Abbildung 6). Zu beachten ist, dass viele dieser Tännchen sehr tief unten einen Trieb aufrichteten und deshalb in Abbildung 6 optisch kleiner erscheinen (vgl. auch Abbildung 7). Unter Berücksichtigung der Ausgangshöhe im statistischen Modell (oder der Höhe im Frühling 2010 nach dem Verbiss) konnten die Tännchen, die einen vorhandenen Trieb aufrichteten, den durch Verbiss bedingten Höhenverlust also kompensieren. Bei allen anderen Reaktionsarten waren die Tännchen im Vergleich zu unverbissenen Bäumchen stets signifikant kleiner: aus basalen Knospen reagierende um $12.5 \pm 6.5 \mathrm{~cm}$, aus distalen Knospen reagierende um $14.7 \pm 7.8 \mathrm{~cm}$; nicht reagierende Tännchen waren im Sommer 2013 mit durchschnittlich $15.1 \pm 6.5 \mathrm{~cm}$ Höhe nur etwa halb so gross wie unverbissene (Abbildung 6).
Der Höhenzuwachs in der ersten Vegetationsperiode nach dem Verbiss im Winter 2009/2010 war bei verbissenen Tännchen kleiner als bei unverbissenen. Schon im zweiten Jahr nach Verbiss war der Höhenzuwachsunterschied jedoch nicht mehr signifikant; unverbissene und verbissene Tännchen wuchsen demzufolge gleich gut, d.h., verbissene kompensierten partiell. Hingegen war der Höhenzuwachs von verbissenen Tännchen 2013, also vier Vegetationsperioden nach Verbiss, im Vergleich zu unverbissenen bei allen Reaktionsarten kleiner (Abbildung 6). Tännchen, welche nach dem Verbiss im Winter 2009/2010 erneut verbissen wurden, wurden von dieser statistischen Analyse ausgeschlossen. Dies dürfte, zusammen mit der um drei bis vier Jahre verzögerten Reaktion einiger Tännchen, den Unterschied im Höhenzuwachs zwei und vier Jahre nach Verbiss erklären (vgl. mehr Mehrfachverbiss bei wenig Licht in Abbildungen 5d und 7). Je weiter oben das Tännchen reagierte, desto grösser war tendenziell auch der Höhenzuwachs; zum Beispiel betrug der durchschnittliche Zuwachs der Tännchen, welche aus einer Knospe basal aus dem 2006/2007 gebildeten Quirl reagiert hatten, $2.4 \pm 0.8 \mathrm{~cm}$, derjenige der Tännchen, welche basal aus einem 2007/2008erQuirl reagiert hatten, $3.6 \pm 2.2 \mathrm{~cm}$ und $5.0 \pm 2.1 \mathrm{~cm}$ bei jenen, die basal aus dem 2008/2009er-Quirl reagierten (vgl. auch Abbildungen 3c und 3d). Generell gilt: je höher der Baum, desto länger sein Höhenzuwachs vor und nach Verbiss.

\section{Wie reagierten die Tännchen auf mehrmaligen Verbiss?}

28 Tännchen wurden in den Flächen A und B zwei- oder dreimal am Endtrieb verbissen, wobei die meisten basal reagierten, gefolgt von keiner und von distaler Reaktion (Tabelle 2 und $\times$ in Abbildung 7). Auffallend ist, dass rund ein Fünftel der mehrmals verbissenen Bäumchen im Juli 2013 noch keinen neuen Endtrieb hatten (Tabelle 2). Nur zwei Tännchen der Flächen A und B richteten nach mehrmaligem Verbiss einen Trieb auf (die rotbraunen $x$ in Abbildung 7), in Fläche C kam das Aufrichten von bestehenden Trieben nicht vor. Zwischen den verschiedenen Reaktionsarten konnten nach mehrmaligem Verbiss weder für die Höhe der Tännchen noch für deren Zuwächse signifikante Unterschiede gefunden werden, wahrscheinlich aufgrund der geringen Anzahl und der wiederum grossen Variabilität (Abbildung 7).

\section{Mehrstämmigkeit}

Lediglich 3.4\% der Bäumchen, die nie einen Endtriebschaden erlitten, hatten zwei oder mehr Endtriebe (Abbildung 8a). Dagegen hatten rund 90\% der mehrstämmigen Tännchen mindestens einmal einen Verlust am Endtrieb, unabhängig von der Fläche (Abbildung 8a) und den Lichtverhältnissen. 

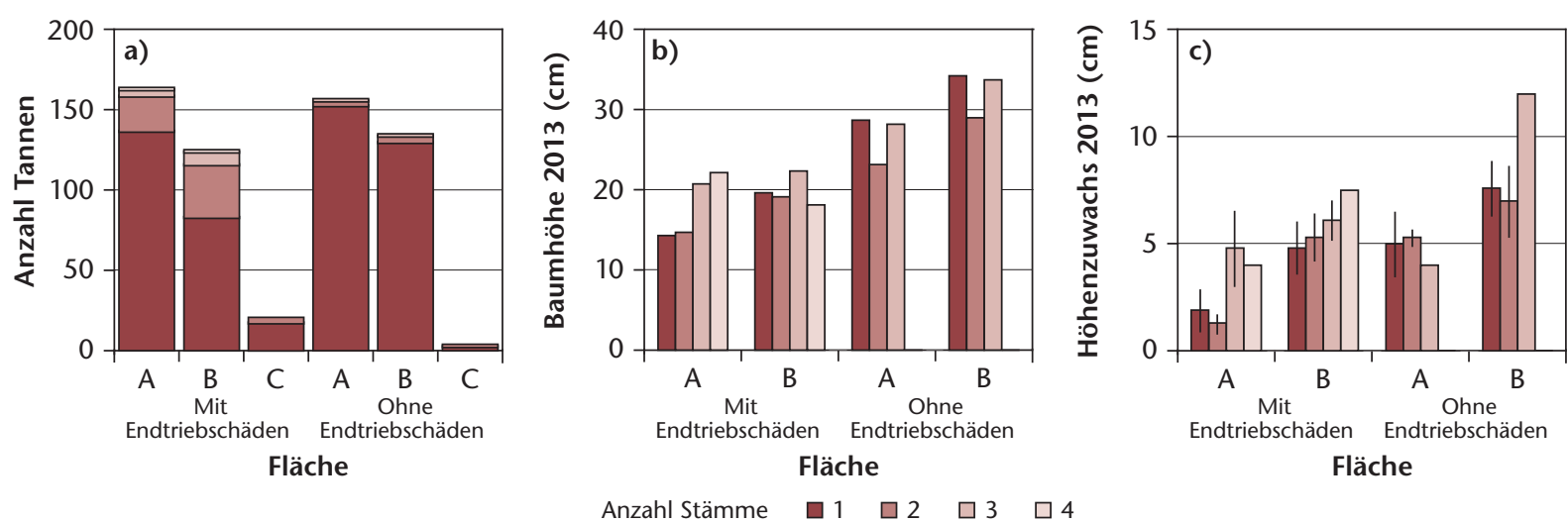

Fläche

Abb 8 Anzahl (a), Baumhöhe 2013 (b) sowie Höhenzuwachs 2013 (c) für ein- und mehrstämmige Weisstännchen, aufgeteilt in solche mit und ohne Endtriebschaden je Fläche. Für den Höhenzuwachs sind jeweils der Mittelwert und die Standardabweichung aufgeführt.

Bei Bäumchen in den Flächen A und B ohne Schaden am Endtrieb waren 2-gipflige im Vergleich zu 1-gipfligen Tännchen signifikant kleiner (Abbildung 8b). Im Gegensatz dazu waren bei Tännchen mit Endtriebschaden die 2- und 3-gipfligen signifikant grösser als die 1-gipfligen (Abbildung 8b), und 3 -gipflige mit Endtriebschaden hatten auch einen signifikant grösseren Höhenzuwachs (Abbildung 8c). Tännchen, die zu Mehrstämmigkeit nach Endtriebverlust neigen, sind also kräftiger mit grösserem Streckungswachstum als solche, die eingipflig bleiben.

\section{Diskussion}

\section{Verbiss}

Nur Gamswild, aber keine Rothirsche oder Rehe verbissen die Tannensämlinge in diesem Experiment. Rehe scheinen die Gegend im Winter zu meiden, wohl wegen der Schneedecke und der Anwesenheit der anderen zwei Wildarten. Grösserer Tannenaufwuchs war hingegen für Hirsche attraktiv, da diese circa 20 bis $100 \mathrm{~cm}$ grosse Tannen in älteren, zur selben Zeit geöffneten Flächen direkt daneben verbissen (vgl. Filme Kupferschmid \& Wasem 2014 und Kupferschmid et al, in Vorbereitung). Generell werden gepflanzte, alleinstehende, kräftigere und ausgemähte Bäumchen häufiger und stärker verbissen als Naturverjüngung derselben Baumart (z.B. Reimoser \& Gossow 1996).

Lokale Muster in der Verbisshäufigkeit konnten gut mit der Schneedauer, die mit dem unbedeckten Himmelsanteil korreliert ist, erklärt werden. Dies erstaunt nicht, da der Verbiss nicht schon im Herbst erfolgte, sondern erst im Winter. Auch Odermatt (2014) fand nur vereinzelt Verbiss an Tannen im Frühwinter, jedoch häufig vor allem im Spätwinter. Ebenso wurden in anderen Studien früher ausapernde Baumgruppen durch Wild öfter verbissen als später ausapernde (Kupferschmid \& Bugmann 2005, Stöckli 2002). Dies hilft eventuell zu erklären, warum der Verbiss an Tannenverjüngung in einigen
Studien mit zunehmendem Licht abnimmt (Senn et al 2007). Dafür verzeichneten wir im Spätwinter 2010 mehr Mausfrass in der Lücke als unter Schirm. Es ist bekannt, dass Mäuseschäden hauptsächlich auf unbeschirmten, vergrasten Flächen auftreten (Odermatt \& Wasem 2004). Dies bedeutet, dass Verbiss entlang des ganzen Lichtgradienten stattfindet, auch wenn nicht zwingend durch dieselben Tierarten. Auch entlang von Lichtgradienten von geschlossenen Wäldern bis in Lotharflächen (1-65\% unbedeckter Himmelsanteil) war die Verbisshäufigkeit an Tännchen unabhängig vom Licht (Kupferschmid et al 2013). Damit fanden wir keine Evidenz dafür, dass gewisse Lichtbedingungen zu einer geringeren Verbissintensität führen würden.

\section{Mortalität}

In den ersten zwei Vegetationsperioden nach der Pflanzung starben die Tännchen ausschliesslich infolge Vertrocknung. In der Nähe unserer Zäune überlebten gesäte und gepflanzte Tännchen besser unter einem Drahtgitterkorb mit $2 \mathrm{~mm}$ Maschenweite als ohne, wahrscheinlich aufgrund der feuchteren Umweltbedingungen (Wasem \& Senn 2000). Im Allgemeinen sind mässig feuchte Bedingungen für die Tannenetablierung am besten (Muscolo et al 2010).

In den Folgejahren beeinflusste der Wasserhaushalt des Bodens die Sterblichkeit jedoch kaum mehr; sind die Pflanzen einmal etabliert, scheint die Mortalität hauptsächlich tierbedingt zu sein. Wiederholter Verbiss erhöht die Sterberate. Natürlich ist zu beachten, dass unsere Resultate primär für Verbiss durch Gämsen gelten, Rehverbiss würde eventuell zu einem anderen Ergebnis führen (vgl. Kupferschmid et al 2013). Im Bayerischen Wald summierte sich die Sterberate über 20 Jahre auf $98 \%$ im geschlossenen Wald, wobei tierbedingt rund 78\% der totalen Verjüngung abstarben (Ammer 1996). Unsere Werte in der überschirmten Fläche C gehen in dieselbe Richtung, über längere Zeit ist daher mit dem Totalausfall der ungeschützten Tännchen zu rechnen. 
Ein Grossteil der toten Tännchen hatte im Vergleich zu den überlebenden entweder sehr wenig oder mehr Licht, d.h., sie befanden sich an einem der Enden des Lichtgradienten in Fläche A (Abbildung 4). Da die Weisstanne eine sehr schattentolerante Baumart ist, erstaunt dieses Resultat nicht. Ab einem unbedeckten Himmelsanteil von 15-16\% ist die Weisstanne offenbar weniger konkurrenzfähig, wird von anderen Pflanzenarten zum Teil überwachsen und riskiert in Föhntälern wie dem Glarnerland in den ersten Monaten (eventuell Jahren) zu vertrocknen oder zusätzlich zum Wild auch noch durch Mäuse gefressen zu werden. Mehr Licht und Konkurrenz durch Bodenvegetation führten auch in anderen Studien zu erhöhter Sämlingssterblichkeit (z.B. Ameztegui \& Coll 2013). Ein unbedeckter Himmelsanteil von unter circa 10\% scheint jedoch sogar für die Tännchen zu dunkel, weshalb dort nur sehr wenige Bäumchen einen (wiederholten) Verbiss überlebten.

Zusammenfassend kann gesagt werden, dass die Überlebenswahrscheinlichkeit der Tännchen unter leichter Beschattung am höchsten ist und jahrelanger, starker Verbiss zum Totalausfall der Tanne führt.

\section{Häufigkeit der Reaktionsarten nach Verbiss}

Die meisten Tännchen bildeten im ersten Jahr nach Verbiss eine neue basale Knospe bei einem Astquirl, verlängerten diese im selben Jahr zu einem Trieb von rund $0.5 \mathrm{~cm}$ Länge und bildeten erst im zweiten Jahr einen eigentlichen neuen Endtrieb. Die Endtriebbildung aus basalen Knospen war auch die häufigste Reaktionsart in Triebschnittexperimenten unter künstlicher Beschattung (Häsler et al 2008). Für die Pflanzenreaktion ist bedeutend, ob am Reststück des verbissenen Endtriebes noch Knospen zurückbleiben, die einen distalen Trieb bilden können, oder ob infolge von zu wenig Licht (Kupferschmid \& Bugmann 2013), zu geringer Grösse des Baumes (Kupferschmid et al 2013) und/oder zu starkem Verbiss (Kupferschmid et al, in Vorbereitung) keine Knospen mehr vorhanden sind. Keine geeigneten Knospen zusammen mit grossem Verlust an Ressourcen helfen auch zu erklären, warum einige unserer Tännchen über zwei bis vier Jahre gar keinen neuen Endtrieb bildeten (Tabelle 2). Keine oder eine verzögerte Reaktion in den ersten Jahren nach einbis mehrmaligem Endtriebverlust scheinen unter künstlicher Beschattung (Häsler et al 2008), bei unterdrückten Tännchen (Kupferschmid \& Bugmann 2013) wie auch im geschlossenen Wald (Kupferschmid et al 2013) besonders häufig zu sein.

Das Aufrichten eines Seitentriebes in Triebschnittexperimenten kam häufiger im Licht als unter künstlicher Beschattung vor (Häsler et al 2008). Im Gegensatz dazu konnte entlang natürlicher (Licht-)Gradienten von geschlossenem Bestand bis
Lotharflächen kein solcher Zusammenhang gefunden werden (Kupferschmid et al 2013). In unserer Studie reagierten zwar $4.5 \%$ der Tännchen mit Aufrichten eines Triebes (Tabelle 2), aber diese waren über den ganzen Lichtgradienten verteilt (Abbildung 7). Zwei Faktoren sind wahrscheinlich für diese Unterschiede verantwortlich: 1) In den Triebschnittexperimenten wurden grosse Teile des Endtriebes, nicht aber der Seitentriebe von grösseren Tännchen abgeschnitten (vgl. Kupferschmid \& Bugmann 2013), und damit blieben oberste kräftige Quirläste unversehrt, die sich aufrichten konnten. Hingegen waren entlang natürlicher Lichtgradienten meist mindestens auch die obersten Seitentriebe verbissen (hier und in Kupferschmid et al 2013), und/oder es wurden nur die Endtriebknospen durch Rehe abgefressen. Im letzteren Fall blieben Knospen am Reststück zurück, aus denen distale Endtriebe austrieben (Kupferschmid et al 2013). 2) In den genannten Triebschnittexperimenten wuchsen insbesondere die Quirläste nicht ganz horizontal, sondern leicht nach oben geneigt. Damit muss zum Aufrichten eines Triebes weniger Druckholz gebildet werden, als wenn die Bäume extrem plagiotrop wachsen. In unserem Versuch wuchsen die dünnen Ästchen aus dem Keimling/2007-Quirl und am 2007 gebildeten Stammstück z.T. auch nicht horizontal (vgl. Abbildung 3) und bildeten deswegen vereinzelt ohne Endtriebschaden einen Zwiesel (Abbildung 8). Eventuell ist Letzteres von der Provenienz der Samen abhängig (Commarmot 1995). Auf jeden Fall hängt die Reaktion von der Verbissstärke ab, und in Gebieten mit viel Verbiss sind Tannenprovenienzen mit gutem Höhenzuwachs, vielen Knospen und nur leichter Plagiotropie ein Vorteil.

Dies bedeutet, dass die Reaktion der Tännchen nach Verbiss, abgesehen von sehr dunklen Beständen, in denen eine verzögerte Reaktion häufig ist, weniger von Standortbedingungen als viel stärker von der Häufigkeit und Stärke des Verbisses und von den verbleibenden intakten Knospen abhängig ist.

\section{Höhe und Zuwachs je Reaktionsart}

Das Aufrichten von Seitentrieben führte zur Kompensation des verbissbedingten Höhenverlustes. Dies scheint logisch, da Tännchen längere Seiten- als Endtriebe besitzen (vgl. Abbildung 3a) und die längeren Seitentriebstücke den Höhenverlust wettmachen, wenn sie sich aufrichten und der Baum aus ihnen direkt weiterwachsen kann. Auch in Tannen-Triebschnittexperimenten und in Studien mit Fichten war Aufrichten die erfolgreichste Reaktionsart (Häsler et al 2008, Welch et al 1992).

Über alle Reaktionsarten gesehen waren aber bereits einmal stark verbissene Tännchen kleiner als unverbissene, und mehrfach stark verbissene noch kleiner (Abbildung 5a), weil eben die allermeisten Tännchen nicht mit Aufrichten, sondern mit neuen 
Trieben aus basalen und distalen Trieben reagierten. Der Höhenzuwachs war nach Verbiss höchstens gleich gross, wohl einfach weil der Zuwachs vorwiegend von der Baumhöhe abhängt und weder von mehr als einem Jahr zurückliegenden Verbissereignissen noch vom Baumalter (vgl. auch Mitscherlich \& Weise 1982).

Verbiss führte in den meisten Fällen zu Mehrstämmigkeit. Damit kommt es wohl nicht nur zur Einbusse in der Höhe, sondern auch in der Qualität (Mitscherlich \& Weise 1982). Es gilt allerdings zu beachten, dass sich die Qualitätseinbussen bei den Tannen im Laufe der Zeit vermindern (Eiberle 1978), zum Beispiel indem die Tannen später wieder einstämmig werden (wie dicht wachsende Fichten, vgl. Welch et al 1992). Die Tatsache, dass mehrgipflige, verbissene Tännchen höher waren und z.T. auch längere Höhenzuwächse hatten als eingipflige, verbissene Tännchen (Abbildung 8), zeigt, dass eine Tanne umso mehr und längere Triebe nach Endtriebverlust bildet, je vitaler sie ist.

Zusammenfassend gibt es spezielle Konstellationen, die zur Höhenkompensation nach einmaligem Verbiss führen, meistens wachsen aber die Tannen zumindest im ersten Jahr nach starkem Verbiss schlechter, und der Höhenunterschied zu unverbissenen Tännchen nimmt mit jedem Verbissereignis zu.

\section{Gibt es einen Licht-Schwellenwert für gute Reaktion?}

Wie mehrere Studien zeigen, wachsen Tannen bei zunehmendem Licht anfänglich besser und erreichen rasch ein Maximum, wonach das Höhenwachstum stagniert oder sogar zurückgeht (Kučeravá et al 2013, Robakowski et al 2003). Sowohl in dieser Studie (Abbildung 5) wie auch auf anderen Schweizer Voralpenstandorten (Napf, Zürcher Oberland) scheint ab ungefähr 11\% unbedecktem Himmelsanteil «guter» Zuwachs möglich. Tendenziell haben aber Tännchen unter besseren Bedingungen mehr Knospen (Kupferschmid et al, in Vorbereitung) und erreichen schneller eine Höhe, bei der sie mit distalen Trieben reagieren können (Kupferschmid et al 2013). Bei demselben Lichtwert von 11\% scheint auch die tier- und umweltbedingte Mortalität gering (Abbildung 4).

\section{Schlussfolgerungen}

Zu dunkle Bestände sind weder für das Höhenwachstum noch für das Überleben und die Reaktion nach Endtriebschädigung der Tanne förderlich. Viel Licht bringt für das Höhenwachstum und die Reaktion nach Verbiss wenig, kann sogar die Sterberate erhöhen und führt zu stärkerer Konkurrenz durch andere Pflanzenarten. Leichte Beschattung ist also das Beste für das Gedeihen der Tannenverjüngung, sei es mit oder ohne Verbiss.
Die Weisstanne ist grundsätzlich fähig, den durch Verbiss verursachten Höhenzuwachsverlust zu kompensieren, aber nur unter gewissen Voraussetzungen. Die Reaktionsfähigkeit hängt insbesondere von den betroffenen Pflanzenteilen ab - also der Verbissstärke (Schädigungsgrad sensu Pollanschütz 1995), d.h. davon, ob nur die Endknospen und der Höhenzuwachs fehlen oder ob zusätzlich auch die Seitentriebe betroffen sind - und von der Knospenzahl am Rest des Baumes. Das Licht spielt vor allem eine Rolle, indem das Wachstum bis zu einem unbedeckten Himmelsanteil von circa 11\% zunimmt und die Knospenzahl mit zunehmendem Licht ansteigt. Da zudem der Verbiss entlang des gesamten Lichtgradienten in etwa gleich ist, empfehlen wir, die Tanne unter leichter Beschirmung zu verjüngen.

Eingereicht: 29. Januar 2014, akzeptiert (mit Review): 14. Mai 2014

\section{Dank}

Wir danken dem Bundesamt für Umwelt für die finanzielle Unterstützung unserer Forschung im Projekt «Langfristige Walddynamik unter Ungulaten-Einfluss» an der ETH Zürich und im Projekt «Auswirkung von Schalenwildverbiss auf Verjüngungsstrukturen: Analysen von Daten aus langjährigen Erhebungen auf Ebene Probeflächen» an der Eidgenössischen Forschungsanstalt WSL. Herzlichen Dank auch an M. Späni, U. Wasem, O. Odermatt und dem Forstpersonal Glarus Süd für deren Einsatz im Feld.

\section{Literatur}

AMEZTEGUI A, COLL L (2013) Unraveling the role of light and biotic interactions on seedling performance of four Pyrenean species along environmental gradients. For Ecol Manage 303: 25-34.

AMMER C (1996) Konkurrenz um Licht zur Entwicklung der Naturverjüngung im Bergmischwald. Weihenstephan: Techn Univ München, Forstl Forschungsber 158. 198 p.

CIOLDI F, BALTENSWEILER A, BRÄNDLI UB, DUC P, GINZLER C ET AL (2011) Waldressourcen. In: Brändli UB, editor. Schweizerisches Landesforstinventar. Ergebnisse der dritten Erhebung 2004-2006. Birmensdorf: Eidgenöss Forsch.anstalt WSL. pp. 31-113.

COMMARMOT В (1995) Internationaler Weisstannen-Herkunftsversuch: Entwicklung der Herkünfte bis zum Alter 12 auf der Versuchsfläche Bourrignon im Schweizer Jura. In: Eder W, editor. Ergebnisse des 7. IUFRO Tannen-Symposiums der WP S. 1.01-08, «Ökologie und Waldbau der Weisstanne». Mainz: IUFRO. pp. 59-68.

EIBERLE K (1978) Folgewirkungen eines simulierten Wildverbisses auf die Entwicklung junger Waldbäume. Schweiz Z Forstwes 129: 757-768.

EIBERLE K, DÜRR C (1985) Grenzen der Verbissbelastung für die Weisstanne (Abies alba) in der kollinen Stufe. Waldhygiene 16: 95-106.

EIBERLE K, NIGG H (1987) Grundlagen zur Beurteilung des Wildverbisses im Gebirgswald. Schweiz Z Forstwes 183: 747-785. 
FREHNER M, WASSER B, SCHWITTER R (2005) Nachhaltigkeit und Erfolgskontrolle im Schutzwald. Wegleitung für Pflegemassnahmen in Wäldern mit Schutzfunktion. Bern: Bundesamt Umwelt Wald Landschaft. $564 \mathrm{p}$.

HÄSLER H, SENN J, EDWARDS PJ (2008) Light-dependent growth responses of young Abies alba to simulated ungulate browsing. Fun Ecol 22: 48-57.

KUČERAVÁ B, DOBROVOLNÝ L, REMEŠ J (2013) Responses of Abies alba seedlings to different site conditions in Picea abies plantations. Dendrobiology 69: 49-58.

KUPFERSCHMID AD, BUGMANN H (2005) Effect of microsites, logs and ungulate browsing on Picea abies regeneration in a mountain forest. For Ecol Manage 205: 251-265.

KUPFERSCHMID AD, BRANG P (2010) Praxisrelevante Grundlagen: Zusammenspiel zwischen Wild und Wald. In: Baumann $M$, Brang P, Burger T, Eyholzer R, Herzog S et al. Wald und Wild Grundlagen für die Praxis. Bern: Bundesamt Umwelt. pp. 9-39.

KUPFERSCHMID AD, BUGMANN H (2013) Timing, light availability and vigour determine the response of Abies alba saplings to leader shoot browsing. Eur J For Res 132: 47-60.

KUPFERSCHMID AD, ZIMMERMANN S, BUGMANN H (2013) Browsing regime and growth response of naturally regenerated Abies alba saplings along light gradients. For Ecol Manage 310: 393-404.

KUPFERSCHMID AD, WASEM U (2014) Filme zum Verbiss an Weisstannen. http://dx.doi.org/10.3929/ethz-a-010075029

KUPFERSCHMID AD, WASEM U, BUGMANN H (2014) Light availability and ungulate browsing determine growth, height and mortality of Abies alba saplings. For Ecol Manage 318: 359-369.

MITSCHERLICH G, WEISE U (1982) Die Fichten-Hemmungsversuche in Abtsgmünd (Fi 304) und Crailsheim (Fi 348). Allg ForstJagdztg 153: 97-104.

MUSCOLO A, SIDARI M, BAGNATO S, MALLAMACI C, MERCURIO R (2010) Gap size effects on above- and below-ground processes in a silver fir stand. Eur J For Res 129: 355-365.

ODERMATT O, WASEM U (2004) Mäuseschäden bei der Wiederbewaldung von Windwurfflächen. Birmensdorf: Eidgenöss Forsch.anstalt WSL. 3 p. www.waldwissen.net/waldwirtschaft/schaden/nager/wsl_maeuseschaeden_windwurfflaechen/wsl_maeuseschaeden_windwurfflaechen_originalversion.pdf (19.5.2014)

\section{Reaktion von Weisstannen auf ein- bis mehr- maligen Verbiss entlang von Lichtgradienten}

Der Verbiss der Weisstanne (Abies alba) durch Schalenwild ist eine der wichtigsten Ursachen für die stark abnehmende Verjüngung dieser Baumart. Die Fähigkeit eines Baumes, auf Verbiss zu reagieren, ist nicht nur genetisch bestimmt, sondern hängt auch von Umweltfaktoren ab. Deshalb untersuchten wir anhand eines Zaunexperiments den Einfluss von natürlichem Verbiss auf das Wachstum von Tännchen entlang eines Lichtgradienten. Vier Vegetationsperioden nach dem Verbiss hatten die wenigen verbissenen Tännchen, welche mit dem Aufrichten eines Triebes reagiert hatten, den verbissbedingten Höhenunterschied im Vergleich zu unverbissenen Bäumchen kompensiert. Die meisten Tännchen hatten aber erst neue Triebe gebildet und waren deshalb kleiner. Je öfter der Endtrieb beschädigt wurde, desto kleiner blieb auch das Tännchen. Bis zu einem unbedeckten Himmelsanteil von zirka 11\% nahm die Baumhöhe linear mit mehr Licht zu. Unter helleren Lichtbedingungen und für den jährlichen Höhenzuwachs war die Korrelation mit Licht weniger deutlich. Die Sterberate war über die gesamten fünf Jahre sowohl bei wenig Licht ( $<10 \%)$ wie auch in Baumlücken (>15\%) hoch infolge Austrocknung, Vegetationskonkurrenz oder Verbiss ( $50 \%$ der Mortalität). Wir empfehlen, die Tannen unter leichter Beschattung zu verjüngen.
ODERMATT O (2009) Ergebnisse aus Untersuchungen zum Wildtiereinfluss auf die Waldverjüngung in der Schweiz. Schweiz Z Forstwes 160: 294-302. doi: 10.3188/szf.2009.0294

ODERMATT O (2014) Wildverbiss: Wann sind die kritischen Phasen? Wald Holz 95 (2): 23-26.

O'REILLY-WAPSTRAA JM, MOOREC BD, BREWERD M, BEATONE J, SIME D ET AL (2014) Pinus sylvestris sapling growth and recovery from mammalian browsing. For Ecol Manage 325: 18-25. POLLANSCHÜTZ J (1995) Bewertung von Verbiss- und Fegeschäden: Hilfsmittel und Materialien. Wien: Forstl Bundesversuchsanstalt, Mitteilungen 169. $146 \mathrm{p}$.

R CORE TEAM (2013) R: A language and environment for statistical computing. Vienna: R Foundation for Statistical Computing.

REIMOSER F, GOSSOW H (1996) Impact of ungulates on forest vegetation and its dependence on the silvicultural system. For Ecol Manage 88: 107-119.

ROBAKOWSKI P, MONTPIED P, DREYER E (2003) Plasticity of morphological and physiological traits in response to different levels of irradiance in seedlings of silver fir (Abies alba Mill.). Trees 17: 431-441.

SENN J, HÄSLER H, BRANG P, ZINGG A (2007) Verbiss der Weisstanne durch Huftiere: Vom Kleinstandort beeinflusst. Wald Holz 88 (1): 39-41.

STAUFFER HB (2008) Contemporary bayesian and frequentist statistical research methods for natural resource scientists. Hoboken NJ: John Wiley. 400 p.

STÖCKLI V (2002) Die Bedeutung der winterlichen Schneedecke für junge Fichten im Gebirgswald: Ergebnisse aus dem Lusiwald bei Davos. Bauhinia 16: 23-32.

TINNER W, ALLGÖWER B, AMMAN B, CONEDERA M, GOBET E ET AL (2005) Ausmass und Auswirkungen der Waldbrände auf die Vegetation der Schweiz im Laufe der Jahrtausende. Schweiz Z Forstwes 156: 325-330. doi: 10.3188/szf.2005.0325 WASEM U, SENN J (2000) Fehlende Weisstannenverjüngung: Hohe Schalenwildbestände können die Ursache sein. Wald Holz 81 (9): 11-14.

WELCH D, STAINES BW, SCOTT D, FRENCH DD (1992) Leader browsing by red and roe deer on young Sitka spruce trees in western Scotland. II. Effects on growth and tree form. Forestry 65: 309-330.

\section{Réactions du sapin blanc à l'abroutisse- ment le long d'un gradient de lumière}

L'abroutissement du sapin blanc (Abies alba) par le gibier est I'une des causes majeures de la diminution du rajeunissement de cette espèce. Bien que l'aptitude d'un arbre à réagir à I'abroutissement est majoritairement d'ordre génétique, des facteurs environnementaux jouent un rôle important. Notre étude examine l'effet de l'abroutissement naturel sur la croissance du sapin le long d'un gradient de lumière, en utilisant des clôtures. Quatre périodes de végétation après I'abroutissement, les quelques sapins ayant réagi par redressement de pousses latérales avaient compensé la différence de hauteur par rapport aux arbrisseaux non broutés. Cependant, la majorité des jeunes sapins avaient développé de nouvelles pousses. Ces derniers sont alors restés significativement plus petits et présentaient des pousses finales plus courtes. Plus les dommages aux pousses finales étaient fréquents, plus les arbrisseaux sont restés petits. Une corrélation positive entre la hauteur de l'arbre et la lumière a pu être observée jusqu'à un degré d'ouverture de 11\%. La mortalité sur l'ensemble des cinq années était élevée aux endroits peu lumineux $(<10 \%)$ et en zone dégagée (>15\%) suite à la dessication, la concurrence végétale ou l'abroutissement des abrisseaux (50\% de la mortalité). Nous conseillons de rajeunir le sapin blanc sous conditions légèrement ombragées. 